\title{
From Discovery to Impact - Near Earth Asteroids
}

\author{
Miloš Tichý ${ }^{1,2}$, Michaela Honková ${ }^{1,3}$, Jana Tichá ${ }^{1}$, Michal Kočer $^{1}$ \\ ${ }^{1}$ Klet Observatory, Zátkovo nabřeží 4, CZ-370 01 České Budějovice \\ South Bohemia, Czech Republic \\ ${ }^{2}$ Czech Technical University in Prague, Faculty of Civil Engineering, \\ Department of Advanced Geodesy, Czech Republic \\ ${ }^{3}$ Brno University of Technology, Faculty of Mechanical Engineering, \\ Institute of Mathematics, Czech Republic \\ mtichy@klet.cz
}

\begin{abstract}
The Near-Earth Objects (NEOs) are the most important of the small bodies of the solar system, having the capability of close approaches to the Earth and the chance to collide with the Earth.

We present here the current system of discovery of these dangerous objects, standards for selecting useful and important targets for NEO follow-up astrometry, system of impact probabilities calculations, and also determination of impact site and evacuation area.
\end{abstract}

Keywords: asteroid, Near Earth Object, astrometry, impact probability

\section{Introduction}

Various kinds of small Solar system bodies orbit the Sun. Minor planets and comets are significant members of these Solar system small bodies population. Signs of catastrophic collisions between the small bodies and the Earth can be seen on the Earth's surface. Therefore to avoid further collisions it is necessary to study Near Earth Objects, i.e. small Solar system bodies, whose orbit crosses the orbit of the Earth.

The Near Earth Objects (NEOs) are the closest small neighbours of the Earth. The NEO research is a quickly expanding field of astronomy, important both for solar system science and for protecting human society from minor planets and comets hazard. Near Earth Objects are sources of impact risk and represent usually a low-probability but potentially a very highconsequence natural hazard. Studies of NEOs moreover contribute importantly to our overall understanding of Solar System, its origin and evolution.

By definition, Near Earth Objects (NEOs) are minor planets (asteroids) and comets with perihelion distance less than 1.3 astronomical unit (AU). The vast majority of NEOs are asteroids, referred to as Near-Earth Asteroids (NEAs). NEAs are divided into four groups (Inner Earth Object /IEO/ or Atira, Aten, Apollo, Amor) according to their perihelion distance, aphelion distance and their semi-major axes. The NEA groups are named according to the significant representant of the group - (2062) Aten discovered January 7, 1976; (1862) Apollo discovered in 1932, but then it was lost until 1973; (1221) Amor discovered also in 1932. There are currently nearly 9000 known NEAs (2012 July) [1]. 
The closer passing larger NEAs are called Potentially Hazardous Asteroids (PHAs). PHAs are NEAs whose Minimum Orbit Intersection Distance (MOID) with the Earth is 0.05 AU or less and whose absolute magnitude $(\mathrm{H})$ is 22.0 or brighter, correlating with estimated diameter exceeding 140 meters. There are currently more than 1300 known PHAs (2012 July). However, the most important sub-category of Near Earth Objects are so-called Virtual Impactors (VIs) [2]. Virtual Impactors are objects for which possible impact solutions, nonzero probability of collision for the next 100 years, exist. Probabilities are calculated from observed positions. As new positional observations become available, the object's orbit is improved, uncertainties are reduced, and the impact solutions are most likely ruled out for the future 100 years, the object being eventually removed from the Virtual Impactors list.

\section{NEO Inventory}

The first task of NEO research is to make inventory of the Near Earth Objects population, therefore the main task of the current Near Earth Objects surveys is to contribute to the inventory of population of NEOs, and more specifically, Potentially Hazardous Asteroids (PHAs) and comets that may pose a threat of impact and thus harm to civilization. Among the most prolific NEO surveys belong the Catalina Sky Survey, Pan-STARRS, LINEAR and Spacewatch. After the actual discovery the measured positions are immediately sent to the Minor Planet Center (MPC) of the International Astronomical Union (IAU). Candidates of newly discovered Near Earth Objects are then published on the NEO Confirmation Page of MPC [3]. Using the service of MPC the observers are able to calculate ephemerides for these bodies (i.e. usualy as a table of values that gives the positions of astronomical object in the sky at a given time as if observed from a particular point on the earth's surface - topocentric positions of the object on the sky in given time interval) including uncertainty plots for followup observations. Further observations of discovered objects are crucial necessary for reliable orbit calculation. There is a number of cooperating follow-up observers all over the world targeting NEOs in need of orbital improvement. Follow-up facilities in Europe are improving their capabilities and providing critical longitudinal coverage. In particular, European followup stations often target NEOs discovered the previous night from the South-Western US [4] . This is the work to which the Klet Observatory in South Bohemia greatly contributes to and the 1.06-m KLENOT telescope at Klet is still the largest telescope in Europe used exclusively for NEO follow-up [5]. If the reliable orbit is calculated, information of the newly discovered Near Earth Object is published including all used astrometric observations and orbital elements in Minor Planet Electronic Circular (MPEC) and impact probability is immediately determined by both JPL SENTRY system (NASA NEO Office) $[6,9]$ and NEODYS system (University in Pisa) [7].

If the nonzero probability of impact for the next 100 years is calculated, then it is necessary to acquire additional information about orbit of the object and to determine its physical and chemical characteristics. Furthermore, an object may come close to the Earth repeatedly on its orbit around the Sun. Every approach to the planet changes its orbit and may lead to future impact to the Earth. Possible orbits leading to impact solutions in the future can be determined, outlining a 'keyhole' area of the sky. If the asteroid doesn't pass through the keyhole, we know it will miss the Earth on its future encounter. If the probability of impact steeply rises with newly obtained observations instead of dropping down to zero, is it necessary to activate methods of defence. 


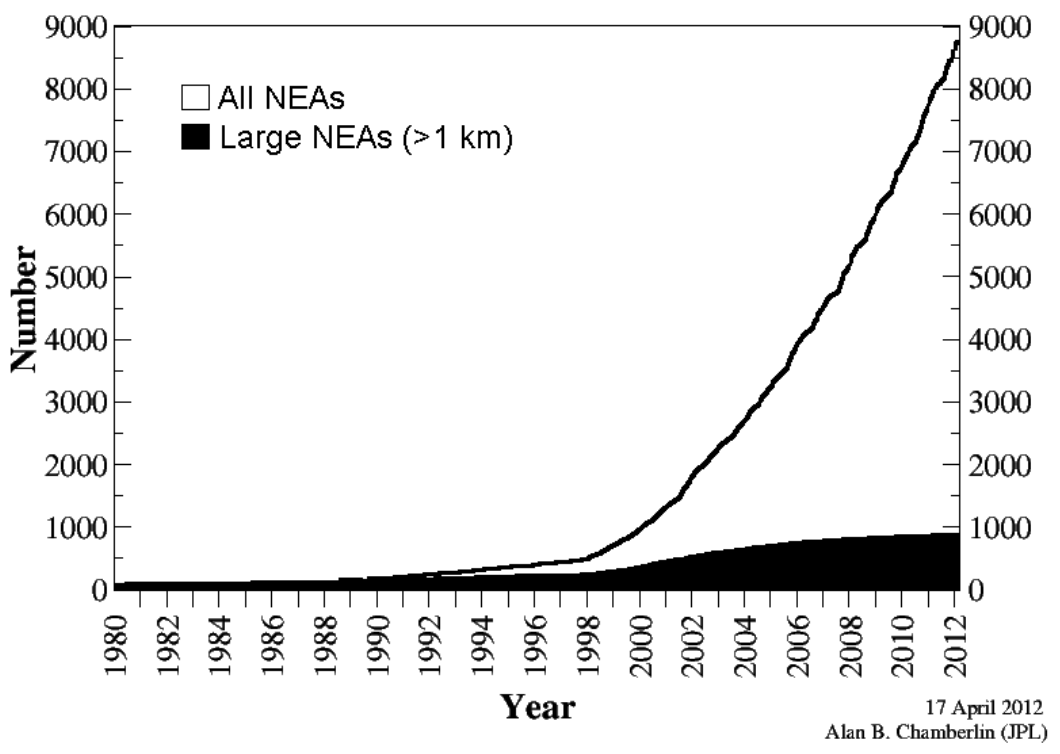

Figure 1: Known Near-Earth Asteroids 1980-January through 2012 March

The Figure 1 shows the cumulative total known near-Earth asteroids versus time. The white area shows all near-Earth asteroids while the black area shows only large near-Earth asteroids (those with diameters roughly one kilometer and larger) [8].

\section{Scenario of impact to the Earth}

The asteroid or comet impact to the Earth has four phases. In the first phase the object travels in the space close to the Earth. Probability of the impact is rising to 1. In the second phase the object enters the Earth's atmosphere. Impact area is determined a few days ahead and was already to be evacuated. The third phase is the impact to the Earth itself. A crater is created and ejecta is flung around. In the last fourth phase shock waves propagate the Earth from the impact area. Energy released and therefore the crater diameter depends on the mass of the body and its velocity. Approximating the mass by object's diameter for an average asteroid density, and assuming common velocity, a $10 \mathrm{~m}$ body has impact energy about 0.06 mega tonnes (Mt) TNT and is capable of local destruction with crater diameter about 300 meters. Larger $100 \mathrm{~m}$ body has at the time of impact an energy of $75 \mathrm{Mt}$ TNT and creates crater 2 kilometres in diameter. That's comparable with the strongest ever detonated nuclear weapon - the Tsar Bomb which had about $50 \mathrm{Mt}$ TNT. One kilometer body would cause an explosion equal to 75 thousands Mt TNT and $11 \mathrm{~km}$ crater with regional to global catastrophe. Energy of impact for a $10 \mathrm{~km}$ body would be 75 millions Mt TNT with a crater $70 \mathrm{~km}$ in diameter and inevitable global catastrophe.

\section{Methods of defence}

NEO impact is the only natural hazard which our civilization can predict and could avoid entirely. Deflection of such a body is feasible - most effective is changing its speed along its path. Disruption following with dispersion are also possible. Finally, complete destruction of the object could also be an option. Deflection is the safest method but requires a warning 
time of a few years to sufficiently change the objects orbit. The second method is disruption following with dispersion, but the asteroid broken into several pieces may just exacerbate the problem. The third option is complete destruction. That requires reliable knowledge regarding its internal structure, rotation, composition etc. Strong enough explosives may not be easily available - to destroy a $100 \mathrm{~m}$ object an energy more than $30 \mathrm{Mt}$ TNT is required, therefore conventional explosives are out of question, and there could arise troubles with acceptability and space transportation of nuclear weapons.

Let's look in detail on deflection methods in the further text. Every scenario can be carried out using either non-nuclear or nuclear methods.

Considering non-nuclear methods, the rotational speed of the object must be taken into account. For fast rotating bodies explosives (standoff, surface or subsurface explosions) and kinetic impactor (similar to impactor probe of comet Tempel 1) can be used. For slowly rotating objects following methods could be considered: focused solar radiation, ion beam, gravity tractor and space tug. The gravity tractor is a spacecraft which relies on the force of gravity between the target asteroid and a spacecraft hovering in close proximity or orbit near it to gradually modify the asteroids orbit. As it uses only gravity force the mechanical composition and structure of the asteroid is not needed to be known. The space tug is similar to the gravity tractor but the spacecraft is physically connected to the asteroid and therefore prior knowledge of surface characteristics would be necessary. For effective deflection both gravity tractor and space tug require long periods of time.

Nuclear methods vary for fast and slow rotators as well. Nuclear explosions (standoff, surface or subsurface) would be effective against fast rotators. For slow rotators gravity tractor or space tug could be used. However use of a nuclear system in space could be a political problem. Use of military technology would be required to save humankind on the Earth, although public could react poorly to it, and a possibility of failure during launch resulting in the bomb exploding on the Earth cannot be rules out [10].

Why is it necessary to have more than one method? Asteroids differ from each other, having different density, size, shape, rotation, some are binary systems etc. and would be discovered with different warning time for an intervention. For each asteroid a different method could be the key to save the Earth [11].

\section{Conclusion}

We presented in this paper the basic parts of the system of principal discovery of potentially dangerous Near Earth Objects, standards for selecting important targets for NEO follow-up astrometry $[12,13]$, system of impact probabilities calculations, and also methods of defense against their devastating impacts.

There are two basic approaches to protect humankind from the NEO hazard.

The first one is a deflection. It is the only responsible way to prevent global disaster to happen. A smaller asteroid discovered shortly before its impact is much more likely scenario to happen than a large asteroid we would know of years ahead, therefore deflection implementation is much less likely than the need to evacuate. Advance decisions must be made before the need to deflect arises. Deflecting an object in space is expensive, especially if an uncertainty in 
its orbit is counted in, resulting in necessity of an additional energy required for successful deflection.

The second approach is an evacuation. It is by far the likely procedure for a last moment response. It is less expensive and more familiar: all usual civil defence emergency procedures can be applied. Evacuation requires rapid, reliable communication between astronomers and officials, and cooperation with emergency managers [14].

Is a collision of asteroid with the Earth just a science-fiction? It is not. We know about many older impact craters on the Earth's surface. For example crater Ries in Bavaria, Barringer crater in Arizona or Tunguska event in 1908. Anyway, the asteroid impact already happened in recent times. The Earth collided with the asteroid 2008 TC3 on 2008 October 7. This object was only 3 meters in diameter and impacted the Nubian desert of Northern Sudan. It was the first and so far the only time the asteroid was observed while still orbiting the Sun, then as a bolide in the Earths atmosphere and finally fragments of the body were found after the impact. So the first part of the NEO "warning" system was already successfully tested $[15]$.

Finally, it is important to say that dealing with the NEO hazard is not a task just for the international scientific community, but it is also the matter for international community as a whole, i. e. governments, relevant decision makers as well as the UN and other relevant international and inter-governmental bodies. The nature and consequences of the threat posed by near Earth asteroid and comet impacts are global and long-term, therefore mitigation efforts will also require coordinated international actions.

Acknowledgement: The work of Klet Observatory and the KLENOT Project is funded by the South Bohemian Regional Authority.

\section{References}

[1] Minor Planet Center of the IAU at http://www.minorplanetcenter.net/

[2] A. Milani, S.R. Chesley, A. Sansaturio, G. Tommei, and G. Valsecchi, "Nonlinear Impact monitoring: Line Of Variation searches for impactors," Icarus, vol. 173, pp. 362-384, 2005.

[3] NEOCP at http://www.cfa.harvard.edu/iau/NEO/ToConfirmRA.html or http:// www . minorplanetcenter.net/iau/NEO/ToConfirmRA.html

[4] S. Larson Current NEO surveys, In: Near Earth Objects, our Celestial Neighbors: Opportunity and Risk, Proc. of IAU Symp. 236. 2007. P. 323 - 328.

[5] J. Ticha, M. Tichy, M. Kocer, and M. Honkova, "KLENOT PROJECT 2002-2008," Meteoritics \& Planetary Science, vol. 44, issue 12, pp. 1889-1895, 2009.

[6] SENTRY at http://neo.jpl.nasa.gov/risk/

[7] NEODYS at http://newton.dm.unipi.it/neodys/

[8] NASA NEO OFFICE at http://neo.jpl.nasa.gov/

[9] A.B. Chamberlin, S.R. Chesley, P.W. Chodas, J.D. Giorgini, M.S. Keesey, R.N. Wimberly, and D.K. Yeomans, "Sentry: An Automated Close Approach Monitoring System for Near-Earth Objects," Bull. Amer. Astron. Soc., vol. 33, pp. 1116, 2001. 
[10] P. Cinzano et al, Europe Mon. Not. R. Astron. Soc. 328, pp. 689-707 (2001)

[11] 2011 IAA Planetary Defence Conference, Bucharest at http://www.pdc2011.org/

[12] J. Ticha, M. Tichy and M. Kocer, The recovery as an important part of NEO astrometric follow-up, Icarus,Vol.159, No. 2, October 2002, pp. 351-357.

[13] D. Koschny, M. Busch, G. Drolshagen, "Asteroid observations at the Optical Ground Station in 2010 - Lessons learnt", 2011 IAA Planetary Defense Conference, Bucharest, 2011

[14] ASE : Asteroid Threats: a Call for Global Response, report to AT-14 at UN-COPUOS 2008 (www.space-explorers.org/committees/NE0/docs/ATACGR.pdf)

[15] Statement of the IAU presented to COPUOS at the 49th session of the Scientific and Technical Subcommittee, Vienna, Austria, 6-17 February 2012. By Karel A. van der Hucht, IAU representative to COPUOS, SRON-Utrecht, the Netherlands 